\title{
Stable transitivity of Euclidean group extensions
}

\author{
IAN MELBOURNE $\dagger \S$ and MATTHEW NICOL $\ddagger$ \\ $\dagger$ Department of Mathematics, University of Houston, Houston, TX 77204-3008, USA \\ (e-mail: i.melbourne@surrey.ac.uk) \\ $\$$ Department of Mathematics and Statistics, University of Surrey, Guildford, \\ Surrey GU2 7XH, UK \\ (e-mail:m.nicol@surrey.ac.uk)
}

(Received 20 January 2001 and accepted in revised form 16 November 2001)

\begin{abstract}
The topological transitivity of non-compact group extensions of topologically mixing subshifts of finite type has been studied recently by Niţică. We build on these methods, and give the first examples of stably transitive non-compact group extensions of hyperbolic dynamical systems. Our examples include extensions of hyperbolic basic sets by the Euclidean group $\mathbf{S E}(n)$ for $n$ even, $n \geq 4$.
\end{abstract}

\section{Introduction}

In this paper, we consider the topological transitivity of non-compact group extensions of hyperbolic basic sets. In particular, we give examples of such extensions that are stably transitive. As far as we know, these are the first examples of non-compact group extensions that are stably transitive. Our results are based upon the work of Niţică [9], which in turn is based upon the work of Brin [2].

Suppose that $F: M \rightarrow M$ is a smooth diffeomorphism of a smooth Riemannian manifold $M$ and that $f: X \rightarrow X$ is the restriction of $F$ to a hyperbolic basic set $X$. Suppose that $\Gamma$ is a finite-dimensional Lie group. Let $r \geq 1$ and define $\mathcal{Z}_{r}$ to be the space of $C^{r}$ maps or cocycles $\zeta: M \rightarrow \Gamma$. Each cocycle $\zeta \in \mathcal{Z}_{r}$ induces a $\Gamma$-extension $f_{\zeta}: X \times \Gamma \rightarrow X \times \Gamma$ given by $f_{\zeta}(x, \gamma)=(f x, \gamma \zeta(x))$.

Definition 1.1. Fix $r \geq 1$ and define

$$
Z=\left\{\zeta \in \mathcal{Z}_{r} \mid f_{\zeta}: X \times \Gamma \rightarrow X \times \Gamma \text { is topologically transitive }\right\} .
$$

If $\zeta \in \operatorname{Int} Z$, then $f_{\zeta}$ is stably (topologically) transitive. If Int $Z$ is dense in $\mathcal{Z}_{r}$, then we say that generically $f_{\zeta}$ is stably transitive. If $Z$ is a residual subset of $\mathcal{Z}_{r}$, then we say that generically $f_{\zeta}$ is transitive.

$\S$ Permanent address: Department of Mathematics and Statistics, University of Surrey, Guildford, Surrey GU2 7XH, UK. 
Let $\mathbf{S E}(n)=\mathbf{S O}(n) \ltimes \mathbb{R}^{n}$ denote the (special) Euclidean group generated by rotations and translations in $n$-dimensional space.

THEOREM 1.2. Let $X$ be a hyperbolic basic set. Suppose that $n$ is even and $n \geq 4$. Then, generically $f_{\zeta}: X \times \mathbf{S E}(n) \rightarrow X \times \mathbf{S E}(n)$ is stably transitive.

More generally, let $\rho: G \rightarrow \mathbf{S O}(n)$ be an orthogonal representation on $\mathbb{R}^{n}$ of a compact connected Lie group $G$. Form the semidirect product $\Gamma=G \ltimes \mathbb{R}^{n}$ with multiplication $\left(g_{1}, v_{1}\right) \cdot\left(g_{2}, v_{2}\right)=\left(g_{1} g_{2}, v_{1}+\rho_{g_{1}} v_{2}\right)$. We call $\Gamma$ a Euclidean-type group.

Our results depend on the following three conditions:

(1) there exists a $g \in G$ such that $I-\rho_{g}$ is a non-singular operator on $\mathbb{R}^{n}$;

(2) $n \geq 2$ and $G$ acts irreducibly on $\mathbb{R}^{n}$;

(3) $G$ is semisimple.

Remark 1.3. When $n$ is odd, condition (1) fails.

Suppose that $\Gamma=\mathbf{S E}(n)$. Then condition (1) holds if and only if $n$ is even, condition (2) holds for all $n \geq 2$ and condition (3) holds if and only if $n \neq 2$.

Theorem 1.2 is an immediate consequence of the next result.

THEOREM 1.4. Let $X$ be a hyperbolic basic set and $\Gamma$ be a Euclidean-type group.

(a) If condition (1) is satisfied, then generically $f_{\zeta}: X \times \Gamma \rightarrow X \times \Gamma$ is transitive.

(b) If conditions (1)-(3) hold, then generically $f_{\zeta}$ is stably transitive.

Remark 1.5. It is an immediate consequence of our work (with $n=0$ ) that extensions of hyperbolic basic sets by compact semisimple Lie groups $G$ are generically stably transitive. In fact, they are stably ergodic (and stably mixing), see [5]. The non-semisimple case requires additional hypotheses on the hyperbolic basic set $[\mathbf{5}, \mathbf{1 2}]$.

Although Euclidean-type groups include $\mathbb{R}^{n}$, our results do not apply to $\mathbb{R}^{n}$-extensions due to the failure of condition (1). Recently, examples of stably transitive $\mathbb{R}^{n}$-extensions (with $X$ Anosov) have been obtained by Niţică and Pollicott [10].

The remainder of this paper is organized as follows. In $\S 2$, we recall the work of Niţică [9] on topological transitivity of skew products and give an elementary proof of Theorem 1.4(a). In $\$ 3$, we prove Theorem 1.4(b).

\section{Transitivity of Euclidean-type group extensions}

This section is divided into three subsections. In §2.1, we recall the work of Niţică [9] on topological transitivity of skew products. In $\$ 2.2$ we collect some elementary results on the structure of Euclidean-type groups. In $\$ 2.3$ we prove Theorem 1.4(a) which gives a sufficient condition for transitivity of Euclidean-type group extensions.

2.1. Topological transitivity of skew products. Suppose that $Y$ is a complete metric space and that $T: Y \rightarrow Y$ is a homeomorphism. A partition $W=\{W(y)\}$ of $Y$ is $T$-invariant if $T(W(y)) \subset W(T(y))$ for all $y \in Y$. A $T$-invariant partition $W$ is contracting if for all $y_{1}, y_{2} \in Y$ with $W\left(y_{1}\right)=W\left(y_{2}\right)$, we have that $d\left(f^{N}\left(y_{1}\right), f^{N}\left(y_{2}\right)\right) \rightarrow 0$ as $N \rightarrow \infty$. The partition is expanding if it is contracting 
for $T^{-1}$. Finally, the partition is of foliation type if given $\epsilon>0$ and any $p \in Y$ and $q \in W(p)$ there exists $\delta(p, q, \epsilon)$ such that if $C$ is a non-empty open set contained in the ball $B(p, \delta)$, then the set $B(q, \epsilon) \cap\left(\bigcup_{z \in C} W(z)\right)$ contains a non-empty open set.

Fix two partitions $W_{1}$ and $W_{2}$ of $Y$. We say that a subset $A \subset Y$ is accessible (with respect to $W_{1}$ and $W_{2}$ ) if for any pair of points $p, q \in A$ there exists $N$ and a chain $\left\{p=y_{1}, y_{2}, \ldots, y_{N}=q\right\}$ such that $y_{i+1} \in W_{1}\left(y_{i}\right) \cup W_{2}\left(y_{i}\right)$ for $i=1, \ldots, N-1$. The subset $A$ is $\epsilon$-accessible if, instead of $y_{N}=q$, we have $d\left(y_{N}, q\right)<\epsilon$.

THEOREM 2.1. (Niţică [9, Theorem 2.1]) Let $Y$ be a complete metric space, $T: Y \rightarrow Y$ be a homeomorphism and $W_{1}, W_{2}$ be a pair of T-invariant partitions of foliation type, one contracting and one expanding. Assume that:

(i) $Y$ is $\epsilon$-accessible for all $\epsilon>0$;

(ii) for any open ball $B \subset Y$ and any positive integer $N$ there is a positive integer $m>N$ such that $T^{m} B \cap B \neq \emptyset$; and

(iii) the conclusion of (ii) holds for $T^{-1}$.

Then $T: Y \rightarrow Y$ is topologically transitive.

Now suppose that $M$ is a smooth Riemannian manifold and let $f: X \rightarrow X$ be the restriction of a smooth diffeomorphism $F: M \rightarrow M$ to a hyperbolic basic set $X$. Let $\Gamma$ be a Euclidean-type group and let $f_{\zeta}: X \times \Gamma \rightarrow X \times \Gamma$ be the $\Gamma$-extension induced by the $C^{r}$ cocycle $\zeta: M \rightarrow \Gamma$.

Since $\Gamma$ admits a left-invariant metric, the $\Gamma$-extension is automatically partially hyperbolic [3] and the stable and unstable partitions $W^{s}$ and $W^{u}$ for $X$ induce (strong) stable and (strong) unstable partitions of foliation type $W^{s s}$ and $W^{u u}$ of $X \times \Gamma$, see [9]. To summarize, we have the following result.

Proposition 2.2. Let $X$ be a hyperbolic basic set and $\Gamma$ be a Euclidean-type group. Then $W^{s s}, W^{u u}$ are a pair of $f_{\zeta}$-invariant partitions of foliation type, one contracting and one expanding.

2.2. Structure of Euclidean-type groups. Let $\Gamma$ be a finite-dimensional Lie group. Suppose that $\gamma_{1}, \ldots, \gamma_{k} \in \Gamma$ and let $\left\langle\gamma_{1}, \ldots, \gamma_{k}\right\rangle$ denote the closed subgroup of $\Gamma$ generated by $\gamma_{1}, \ldots, \gamma_{k}$. Let $\left\langle\gamma_{1}, \ldots, \gamma_{k}\right\rangle^{+}$denote the closed semisubgroup generated by $\gamma_{1}, \ldots, \gamma_{k}$. Define

$$
\begin{aligned}
C & =\{\gamma \in \Gamma:\langle\gamma\rangle \text { is compact }\} \\
F_{k} & =\left\{\left(\gamma_{1}, \ldots, \gamma_{k}\right) \in \Gamma^{k}:\left\langle\gamma_{1}, \ldots, \gamma_{k}\right\rangle=\Gamma\right\} \\
F_{k}^{+} & =\left\{\left(\gamma_{1}, \ldots, \gamma_{k}\right) \in \Gamma^{k}:\left\langle\gamma_{1}, \ldots, \gamma_{k}\right\rangle^{+}=\Gamma\right\} .
\end{aligned}
$$

Note that

$$
C^{k} \cap F_{k} \subset F_{k}^{+} \subset F_{k} .
$$

Now suppose that $\Gamma=G \ltimes \mathbb{R}^{n}$ is a Euclidean-type group. Define $G_{C} \subset G$ to be the set

$$
G_{C}=\left\{g \in G: I-\rho_{g} \text { is a non-singular operator on } \mathbb{R}^{n}\right\} .
$$


PROPOSITION 2.3.

(a) $g \in G_{C}$ if and only if $(g, v) \in C$ for all $v \in \mathbb{R}^{n}$.

(b) If condition (1) holds, then $G_{C}$ is open and dense in $G$, and $C$ contains the open and dense subset $G_{C} \times \mathbb{R}^{n} \subset \Gamma$.

Proof. Compute that $(g, v)^{m}=\left(g^{m}, v_{m}\right)$, where

$$
v_{m}=\left(I+\rho_{g}+\cdots+\rho_{g}^{m-1}\right) v .
$$

If $g \in G_{C}$, then $v_{m}=\left(I-\rho_{g}\right)^{-1}\left(I-\rho_{g}^{m}\right) v$ is bounded and $(g, v) \in C$. Otherwise, choosing $v \in \operatorname{ker}\left(I-\rho_{g}\right)$ yields an element $(g, v) \notin C$. This proves part (a).

To prove part (b), note that $G_{C}$ is a Zariski open subset of $G$. Condition (1) guarantees that $G_{C}$ is non-empty and, hence, open and dense in $G$. By (a), $G_{C} \times \mathbb{R}^{n} \subset C$.

PROPOSITION 2.4. Suppose that $\Gamma$ is a Euclidean-type group. Then $F_{n+3}$ is a residual subset of $\Gamma^{n+3}$.

Proof. We use the well-known facts that:

(i) if $w_{1}, \ldots, w_{n+1} \in \mathbb{R}^{n}$, then generically $\left\langle w_{1}, \ldots, w_{n+1}\right\rangle=\mathbb{R}^{n}$ (cf. [9, Lemma 2.6]); and

(ii) if $g_{1}, g_{2} \in G$, then generically $\left\langle g_{1}, g_{2}\right\rangle=G$ (see [1]).

A standard argument shows that $F_{n+3}$ is a countable intersection of open subsets of $\Gamma^{n+3}$ which is a Baire space, so it suffices to show that $F_{n+3}$ is dense in $\Gamma^{n+3}$. Let $\Sigma=\left\langle\gamma_{1}, \ldots, \gamma_{n+3}\right\rangle$ and let $\Sigma_{2}=\left\langle\gamma_{1}, \gamma_{2}\right\rangle$. We show that $\Sigma=\Gamma$ for a dense subset of $(n+3)$-tuples $\left(\gamma_{1}, \ldots, \gamma_{n+3}\right) \in \Gamma^{n+3}$.

Write $\gamma_{j}=\left(g_{j}, v_{j}\right)$ where $g_{j} \in G, v_{j} \in \mathbb{R}^{n}$. By (ii), we may suppose that $\left\langle g_{1}, g_{2},\right\rangle=G$ and, hence, $\pi\left(\Sigma_{2}\right)$ is dense in $G$. (Without compactness, we cannot directly deduce that $\pi\left(\Sigma_{2}\right)=G$.) Hence, for $j \geq 3$, we can perturb $g_{j}$ to lie in $\pi\left(\Sigma_{2}\right)$. In particular, for each $j \geq 3$, we have two corresponding elements $\gamma_{j}=\left(g_{j}, v_{j}\right)$, $\delta_{j}=\left(g_{j}, w_{j}\right)$ in $\Sigma$ where $w_{j}$ depends only on $\gamma_{1}$ and $\gamma_{2}$. Hence, we obtain pure translations $\left(e, z_{j}\right)=\gamma_{j} \delta_{j}^{-1}, j \geq 3$, where $z_{j}=v_{j}-w_{j}$. The translations $v_{j}, j \geq 3$ are still free to be perturbed, so by fact (i), we may suppose that $z_{3}, \ldots, z_{n+3}$ generate $\mathbb{R}^{n}$, so that $\{e\} \times \mathbb{R}^{n} \in \Sigma$. It follows that $\left(g_{1}, 0\right),\left(g_{2}, 0\right) \in \Sigma$. Hence $G \times\{0\} \subset \Sigma$ and so $\Sigma=\Gamma$.

\subsection{Criteria for transitivity.}

COROLlaRY 2.5. Let $X$ be a hyperbolic basic set and $\Gamma$ be a Euclidean-type group satisfying condition (1). Then generically $f_{\zeta}: X \times \Gamma \rightarrow X \times \Gamma$ is $\epsilon$-accessible for all $\epsilon>0$.

Proof. By Proposition 2.3, we have that $C$ contains an open and dense subset of $\Gamma$. By Proposition 2.4, $F_{n+3}$ is residual. It follows from (2.1) that $F_{n+3}^{+}$is residual. Genericity of $\epsilon$-accessibility is immediate from the proof of [9, Lemma 2.2].

PROPOSITION 2.6. Let $X$ be a hyperbolic basic set and $\Gamma$ be a Euclidean-type group satisfying condition (1). Then generically, $f_{\zeta}: X \times \Gamma \rightarrow X \times \Gamma$ has the property that recurrent points are dense in $X \times \Gamma$. 
Proof. For $N \geq 1, f_{\zeta}^{N}(x, \gamma)=\left(f^{N} x, \gamma \zeta_{N}(x)\right)$, where

$$
\zeta_{N}(x)=\zeta(x) \zeta(f x) \cdots \zeta\left(f^{N-1} x\right) .
$$

If $f^{p} x=x$, then $f_{\zeta}^{p j}(x, \gamma)=\left(x, \gamma \zeta_{p}(x)^{j}\right)$ for all $j \geq 1$. Hence, $(x, \gamma)$ is recurrent if and only if $\zeta_{p}(x) \in C$. It follows from Proposition 2.3 that this is the case for an open and dense set of $\Gamma$-extensions. Since $X$ contains a countable dense set consisting of periodic points, generically recurrent points are dense in $X \times \mathbf{S E}(n)$.

Proof of Theorem 1.4(a). It follows from Proposition 2.2, Corollary 2.5 and Proposition 2.6 that the hypotheses of Theorem 2.1 are generically valid.

\section{Stable transitivity of Euclidean-type group extensions}

In this section we prove Theorem 1.4(b), which gives a sufficient condition for stable transitivity of Euclidean-type group extensions. We require the following two results which strengthen the conclusions of Propositions 2.4 and 2.6, respectively.

THEOREM 3.1. Suppose that $\Gamma$ is a Euclidean-type group satisfying conditions (1)-(3). Then $F_{2}$ is (Zariski) open and dense in $\Gamma^{2}$.

THEOREM 3.2. Let $X$ be a hyperbolic basic set and $\Gamma$ be a Euclidean-type group satisfying condition (1). Then an open and dense set of $\Gamma$-extensions $f_{\zeta}: X \times \Gamma \rightarrow X \times \Gamma$ have recurrent points dense in $X \times \Gamma$.

The proof of part (b) of Theorem 1.4 now follows the proof of part (a). It remains to prove Theorems 3.1 and 3.2. This is done in $\$ 3.1$ and $\$ 3.2$, respectively.

\subsection{Proof of Theorem 3.1.}

THEOREM 3.3. [4, 7] Suppose that $G$ is a compact connected semisimple Lie group. Then $F_{2}$ is a non-empty Zariski open subset of $G^{2}$.

The openness and density of $F_{2}$ is due to Kuranishi [7]. Zariski openness was proved recently by Field [4].

LEMMA 3.4. Let $\Gamma=G \ltimes \mathbb{R}^{n}$ be a Euclidean-type group satisfying conditions (1) and (3) and with projection $\pi: \Gamma \rightarrow G$. Suppose that $\Sigma$ is a finitely generated closed subgroup of $\Gamma$ such that $\overline{\pi(\Sigma)}=G$. Then $\pi(\Sigma)=G$.

Proof. Let $\pi_{*}=(d \pi)_{e}: L \Sigma \rightarrow L G$ be the corresponding homomorphism of Lie algebras. Then it suffices to show that $\pi_{*}$ is onto.

By condition (3), $L G$ can be written uniquely as a direct sum of simple Lie algebras $L G=L_{1} \oplus \cdots \oplus L_{r}$. Moreover, $G$ is a finite cover of a finite product of simple Lie groups $G_{1}, \ldots, G_{r}$ where $L_{j}=L G_{j}$. Let $p_{j}: G \rightarrow G_{j}$ denote the obvious projections and $\pi_{j}=p_{j} \circ \pi: \Sigma \rightarrow G_{j}$. Observe that $\pi_{j}(\Sigma)$ is dense in $G_{j}$.

Let $V=\pi_{*}(L \Sigma)$. Since $\pi(\Sigma)$ is dense in $G$ it follows that $V$ is invariant under the adjoint action of $G$ on $L G$ and so $V$ is an ideal in $L G$. By the semisimplicity 
of $L G$ again, $V$ is a direct sum of some of the factors $L_{1}, \ldots, L_{r}$. Relabelling, we can write $V=L_{1} \oplus \cdots \oplus L_{s}$ where $s \leq r$.

It remains to show that $s=r$. It suffices to show that for each $j$, there is a closed subgroup $T \subset G_{j}$ with $\operatorname{dim} T \geq 1$ such that $T \in \pi_{j}(\Sigma)$. Since $\pi_{j}(\Sigma)$ is a finitely generated group of matrices, Selberg's Lemma [13] (see also [11, p.18]) guarantees that there exists a torsion-free subgroup $N \subset \pi_{j}(\Sigma)$ of finite index. By condition $(1), p_{j}\left(G_{C}\right)$ is open and dense in $G_{j}$ so $N \cap p_{j}\left(G_{C}\right) \neq\{e\}$. In particular, there exists an element $g \in G_{j}$ of infinite order such that the corresponding element $\gamma \in \Sigma$ generates a compact subgroup $\langle\gamma\rangle \subset \Sigma$. Let $T=\pi_{j}\langle\gamma\rangle$. Then $T \subset \pi_{j}(\Sigma)$ is an infinite compact subgroup of $G_{j}$ and so $\operatorname{dim} T \geq 1$ as required.

LEMMA 3.5. Let $\Gamma=G \ltimes \mathbb{R}^{n}$ be a Euclidean-type group satisfying condition (2) and with projection $\pi: \Gamma \rightarrow G$. Suppose that $\Sigma$ is a closed subgroup of $\Gamma$ such that $\pi(\Sigma)=G$. Then either $\Sigma=\Gamma$ or $\Sigma \cong G$.

Proof. Identify $\{e\} \times \mathbb{R}^{n}$ with $\mathbb{R}^{n}$ and let $\mathcal{L}=\Sigma \cap \mathbb{R}^{n}$. Then $\mathcal{L}$ is a closed subgroup of $\mathbb{R}^{n}$ and is given by $\mathcal{L}=\mathbb{R}^{p} \times \mathbb{Z}^{q}$ where $p, q \geq 0$. We claim that $G$ preserves $\mathcal{L}$, so for any $(v, w) \in \mathbb{R}^{p} \times \mathbb{Z}^{q}$ and $g \in G$, we can write $\rho_{g}(v, w)=\left(v_{g}, w_{g}\right) \in \mathbb{R}^{p} \times \mathbb{Z}^{q}$. Since $G$ is connected, $w_{g} \equiv w$ so that $G$ acts trivially on $\mathbb{Z}^{q}$. By condition (2), $\mathbb{Z}^{q}=0$ so that $\mathcal{L}=\mathbb{R}^{p}$. Moreover, $\mathcal{L}=\mathbb{R}^{n}$ or $\mathcal{L}=0$ as required.

To prove the claim, note that for each $g \in G$, there exists a $v_{g} \in \mathbb{R}^{n}$ such that $\left(g, v_{g}+\mathcal{L}\right) \subset \Sigma$ and $v_{g}$ is unique modulo $\mathcal{L}$. We have $\left(g, v_{g}+\mathcal{L}\right)^{2}=\left(g^{2}, v_{g^{2}}+\mathcal{L}\right)$ and so

$$
v_{g^{2}}+\mathcal{L}=\rho_{g}\left(v_{g}+\mathcal{L}\right)+v_{g}+\mathcal{L} .
$$

Let $w=v_{g^{2}}-\rho_{g} v_{g}-v_{g}$. Then $w+\rho_{g} \mathcal{L}=\mathcal{L}$. Since $0 \in \rho_{g} \mathcal{L}$, we deduce that $w \in \mathcal{L}$ and, hence, $\rho_{g} \mathcal{L}=\mathcal{L}$ as required.

Lemma 3.6. Suppose that $\Gamma$ is a Euclidean-type group. Let

$$
A=\left\{\left(\gamma_{1}, \gamma_{2}\right) \in \Gamma^{2}: \overline{\left\langle\gamma_{1}, \gamma_{2}\right\rangle} \text { is not compact }\right\} .
$$

Then A contains a non-empty Zariski open subset of $\Gamma^{2}$.

Proof. For $j=1,2$, write $\gamma_{j}=\left(g_{j}, v_{j}\right)$ where $g_{j} \in G$ and $v_{j} \in \mathbb{R}^{n}$. Define $D=G-G_{C}$ which is Zariski closed in $G$.

Observe that $\gamma_{1}^{n}=\left(g_{1}^{n}, n v_{1}\right)$ for all $v_{1} \in \operatorname{ker}\left(I-\rho_{g_{1}}\right)$. Hence, $\gamma_{1}$ generates a noncompact group for all those $v_{1}$ that have a non-zero component in $\operatorname{ker}\left(I-\rho_{g_{1}}\right)$. It follows that if $D=G$, then $\overline{\left\langle\gamma_{1}\right\rangle}$ is non-compact for a non-empty Zariski open set of $\gamma_{1} \in \Gamma$.

It remains to consider the case when $D \neq G$. Define

$$
A^{\prime}=\left\{\left(\gamma_{1}, \gamma_{2}\right) \in \Gamma^{2}: g_{1}, g_{2} \in G_{C} \text { and }\left(I-\rho_{g_{1}}\right)^{-1} v_{1} \neq\left(I-\rho_{g_{2}}\right)^{-1} v_{2}\right\} .
$$

Clearly, $A^{\prime}$ is Zariski open and non-empty. We show that $A^{\prime} \subset A$.

Suppose that $\Sigma=\overline{\left\langle\gamma_{1}, \gamma_{2}\right\rangle}$ is compact. We must show that $\left(\gamma_{1}, \gamma_{2}\right) \notin A^{\prime}$. The Euclidean-type action of $\Gamma$ on $\mathbb{R}^{n}\left(x \rightarrow \rho_{g} x+v\right)$ restricts to an action of $\Sigma$ on $\mathbb{R}^{n}$. Choose $x_{0} \in \mathbb{R}^{n}$ and define $y=\int_{\Sigma} \sigma x_{0} d \mu$ where $\mu$ is normalized Haar measure on $\Sigma$. Let $\sigma_{0}=\left(g_{0}, v_{0}\right) \in \Sigma$. Then

$$
\sigma_{0} y=\int_{\Sigma} \rho_{g_{0}} \sigma x_{0} d \mu+v_{0}=\int_{\Sigma}\left(\rho_{g_{0}} \sigma x_{0}+v_{0}\right) d \mu=\int_{\Sigma} \sigma_{0} \sigma x_{0} d \mu=y .
$$


Hence, $\Sigma$ fixes the point $y$. In particular, $\gamma_{j} y=y$ for $j=1,2$. This means that $\left(I-g_{1}\right) y=v_{1}$ and $\left(I-g_{2}\right) y=v_{2}$. If $g_{1}, g_{2} \in G_{C}$, then $y=\left(I-g_{1}\right)^{-1} v_{1}=\left(I-g_{2}\right)^{-1} v_{2}$. Hence, $\left(\gamma_{1}, \gamma_{2}\right) \notin A^{\prime}$ as required.

Proof of Theorem 3.1. Let $\Sigma$ denote the closed subgroup of $\Gamma$ generated by $\gamma_{1}$ and $\gamma_{2}$. By conditions (1) and (3), Theorem 3.3 and Lemma 3.4, there is a non-empty Zariski open subset $U \subset \Gamma^{2}$ consisting of pairs $\left(\gamma_{1}, \gamma_{2}\right) \in \Gamma^{2}$ for which $\pi(\Sigma)=G$. By condition (2) and Lemma 3.5, either $\Sigma \cong G$ or $\Sigma=\Gamma$. However, by Lemma 3.6, we can shrink $U$ if necessary so that in addition $\Sigma$ is non-compact and, hence, $\Sigma=\Gamma$.

3.2. Proof of Theorem 3.2. Suppose that $G \subset \mathbf{S O}(n)$ is a compact connected Lie group. Recall that the open set $G_{C}$ consists of those elements of $G$ such that $I-\rho_{g}$ is non-singular.

Let $f: X \rightarrow X$ be a hyperbolic basic set and let $h: X \rightarrow G$. For $x \in X$ and $N \geq 1$, define

$$
h_{N}(x)=h(x) h(f x) \cdots h\left(f^{N-1} x\right)
$$

Suppose that $x \in X$ is a periodic point of period $p$. We say that $x$ is $\operatorname{good}$ if $h_{p}(x) \in G_{C}$. Otherwise $x$ is bad. Our main result in this section is the following.

THEOREM 3.7. Suppose that $h: X \rightarrow G$ is Lipschitz. Either good periodic points are dense in $X$, or all periodic points are bad.

We require the following (strengthened) version of the Anosov closing lemma.

Lemma 3.8. (Anosov Closing Lemma [6, p. 269]) Suppose that $X$ is a hyperbolic basic set. There exist constants $C_{1} \geq 1, \epsilon>0,0<\lambda<1$ such that if $d\left(f^{n}(v), v\right)<\epsilon$ for some $v \in X, n \geq 1$, then there exists $w \in X$ with $f^{n} w=w$ such that

$$
d\left(f^{j} v, f^{j} w\right)<C_{1} \lambda^{\min (j, n-j)} d\left(f^{n} v, v\right),
$$

for $j=1,2, \ldots, n$.

Proof of Theorem 3.7. Suppose that good periodic points are not dense and fix the periodic point $x_{0} \in X$. We show that $x_{0}$ is bad. By passing to a power of $f$, we may suppose without loss that $x_{0}$ is a fixed point. Let $g_{0}=h\left(x_{0}\right)$ and define $D=G-G_{C}$. We show that $g_{0} \in D$.

Since good periodic points are not dense, there is a non-empty open subset $U_{0} \subset X$ such that all periodic points in $U_{0}$ are bad. Let $U=\bigcup_{j \in \mathbb{Z}} f^{j}\left(U_{0}\right)$. Then $U$ is an open dense subset of $X$ and all periodic points in $U$ are bad.

Let $C_{1}, \epsilon, \lambda$ be as in Lemma 3.8. Choose a periodic point $z$ such that:

(i) $z$ and $f z$ are within distance $\epsilon / 2$ of $x_{0}$;

(ii) there is a $j \geq 1$ such that points within distance $C_{1} d(z, f z)$ of $f^{j} z$ lie inside $U$.

(This can be achieved by first fixing $y \in U$ and then shadowing a pseudo-orbit that travels from $x_{0}$ to $y$ and back again to $x_{0}$.) Let $m$ denote the period of $z$ and define $\sigma=h_{m}(z)$. 
Fix $s \geq 1$ and note that $f^{m s+1} z=f z$, which is within a distance $\epsilon$ of $z$. By Lemma 3.8, there exists a periodic point $p$ of period $m s+1$ such that

$$
d\left(f^{j} p, f^{j} z\right)<C_{1} \lambda^{\min (j, m s+1-j)} d(z, f z) \leq C_{1} d(z, f z),
$$

for $j=1,2, \ldots, m s+1$. It follows from property (ii) above that $f^{j} p \in U$ so that $p$ is a bad periodic point. Hence, $h_{m s+1}(p) \notin G_{C}$. By construction $h_{m s+1}(z)=\sigma^{s} h(f z)$.

We claim that there are universal constants $K$ and $\alpha$ (depending only on $f: X \rightarrow X$ and $h: X \rightarrow G)$ such that $\operatorname{dist}\left(\sigma^{s} h(f z), D\right) \leq K d(z, f z)^{\alpha}$. Since $\epsilon$ is arbitrarily small (so the three points $x_{0}, z$ and $f z$ are arbitrarily close to each other), $\sigma^{s} h(f z)$ is arbitrarily close to $D$ and $h(f z)$ is arbitrarily close to $g_{0}$. At the same time, we can choose $s$ so that $\sigma^{s}$ is arbitrarily close to the identity. Altogether, we have that $g_{0}$ is arbitrarily close to the closed set $D$ and, hence, $g_{0} \in D$ as required.

It remains to verify the claim. The proof closely follows the proof of the Livšic Periodic Point Theorem [6, p. 609]. Let $M$ be the Lipschitz constant for $h$. It is easily verified by induction that

$$
h_{N}(x)-h_{N}(y)=\sum_{j=0}^{N-1} h_{j}(x)\left[h\left(f^{j} x\right)-h\left(f^{j} y\right)\right] h_{j}(y)^{-1} h_{N-1}(y),
$$

for all $x, y \in X, N \geq 1$. Hence,

$$
\begin{aligned}
\operatorname{dist}\left(\sigma^{s} h(f z), D\right) & \leq\left\|h_{m s+1}(z)-h_{m s+1}(p)\right\| \leq \sum_{j=0}^{m s}\left\|h\left(f^{j} z\right)-h\left(f^{j} p\right)\right\| \\
& \leq \sum_{j=0}^{m s} M d\left(f^{j} z, f^{j} p\right) \leq M \sum_{j=0}^{m s} C_{1} \lambda^{\min (j, m s+1-j)} d(z, f z) \\
& =M C_{1} d(z, f z) \sum_{j=0}^{m s} \lambda^{\min (j, m s+1-j)} \\
& \leq 2 M C_{1}(1-\lambda)^{-1} d(z, f z) .
\end{aligned}
$$

The claim follows with $K=2 M C_{1}(1-\lambda)^{-1}$.

Proof of Theorem 3.2. Write elements of $\Gamma$ as $\gamma=(g, v)$, where $g \in G$ and $v \in \mathbb{R}^{n}$. Similarly, write $\zeta(x)=(h(x), k(x))$ where $h: X \rightarrow G$ and $k: X \rightarrow \mathbb{R}^{n}$ are the restrictions (from $M$ to $X$ ) of $C^{r}$ maps. The heights $\zeta_{N}(x) \in \Gamma$ and $h_{N}(x) \in G$ (defined in (2.2) and (3.1), respectively) are related by the fact that $h_{N}(x)$ is the $G$-component of $\zeta_{N}(x)($ cf. $[8])$.

Choose a periodic point $x_{0} \in X$. By Proposition 2.3(b), $G_{C}$ is open and dense in $G$, so $x_{0}$ is a good periodic point for an open and dense set of $\Gamma$-extensions $f_{\zeta}$. By Theorem 3.7, good periodic points are then dense in $G$. For such periodic points $x$, with period $p(x)$, we have $h_{p(x)}(x) \in G_{C}$ and, hence, by Proposition 2.3(a) $\zeta_{p(x)}(x) \in C$. As shown in Proposition 2.6, it follows immediately that $(x, \gamma)$ is recurrent in $X \times \Gamma$ for all $\gamma \in \Gamma$. Hence, recurrent points are dense as required. 
Acknowledgements. We are grateful to Andrew Török for pointing out a gap in an earlier draft of the paper. I. Melbourne was supported in part by NSF grant DMS-0071735 and M. Nicol was supported in part by EPSRC grant GR/L98923.

\section{REFERENCES}

[1] H. Auerbach. Sur les groupes linéaire bornés (III). Studia Mat. 5 (1934), 43-49.

[2] M. I. Brin. Topological transitivity of one class of dynamical systems and flows of frames of negative curvature. Funct. Anal. Appl. 9 (1975), 9-19.

[3] M. I. Brin and J. B. Pesin. Partially hyperbolic dynamical systems. Math. USSR Izv. 8 (1974), 177-218.

[4] M. J. Field. Generating sets for compact semisimple Lie groups. Proc. Amer. Math. Soc. 127 (1999), 3361-3365.

[5] M. J. Field and W. Parry. Stable ergodicity of skew extensions by compact Lie groups. Topology 38 (1999), 167-187.

[6] A. Katok and B. Hasselblatt. Introduction to the Modern Theory of Dynamical Systems (Encyclopedia of Mathematics and its Applications, 54). Cambridge University Press, Cambridge, 1995.

[7] M. Kuranishi. Two element generations on semi-simple Lie groups. Kodai Math. Sem. Rep. (1949), 9-10.

[8] M. Nicol, I. Melbourne and P. Ashwin. Euclidean extensions of dynamical systems. Nonlinearity 14 (2001), 275-300.

[9] V. Niţică. Examples of topologically transitive skew-products. Discrete Continuous Dynam. Syst. 6 (2000), 351-360.

[10] V. Niţică and M. Pollicott. Transitivity of Euclidean extensions of Anosov diffeomorphisms. Ergod. Th. \& Dynam. Sys. to appear.

[11] A. L. Onishchik and E. B. Vinberg. Lie Groups and Lie Algebras. II (Encyclopaedia of Mathematical Science, 21). Springer, Berlin, 2000.

[12] W. Parry and M. Pollicott. Stability of mixing for toral extensions of hyperbolic systems. Proc. Steklov Inst. 216 (1997), 354-363.

[13] A. Selberg. On discontinuous groups in higher-dimensional symmetric spaces. Contributions to Function Theory. Bombay, 1960, pp. 147-164. 